\title{
Docosahexaenoic acid sensitizes colon cancer cells to sulindac sulfide-induced apoptosis
}

\author{
SOO-JEONG LIM ${ }^{1}$, EUNMYONG LEE ${ }^{1}$, EUN-HYE LEE ${ }^{1}$, SOO-YEON KIM ${ }^{1}$, JUN HYUNG CHA ${ }^{2}$, \\ HWANHO CHOI $^{1}$, WANSEO PARK ${ }^{3}$, HYEON KYEOM CHOI $^{4}$, SEONG-HEE KO ${ }^{4}$ and SO HEE KIM ${ }^{4,5}$ \\ ${ }^{1}$ Department of Bioscience and Biotechnology, Sejong University, Seoul; ${ }^{2}$ Hyundai High School, Seoul; \\ ${ }^{3}$ Research Institute, National Cancer Center, Goyang, Gyeonggi; ${ }^{4}$ College of Dentistry and Research \\ Institute of Oral Science, Gangneung-Wonju National University, Gangneung, Republic of Korea
}

Received September 18, 2011; Accepted November 28, 2011

DOI: $10.3892 /$ or.2012.1706

\begin{abstract}
Sulindac analogs represent one of the most efficacious groups of NSAIDs reducing the risk of colon cancer. Recent studies have shown that sulindac sulfide, a sulindac analog effective at lower doses compared to its parent compound, triggers the death receptor (DR)5-dependent extrinsic apoptotic pathway. Induction of apoptosis via activation of the DR-mediated pathway would be an ideal therapeutic strategy to eliminate cancer cells. In this study, we investigated the possibility that colon cancer cells are sensitized to sulindac sulfide-induced apoptosis by docosahexaenoic acid (DHA), via activation of the DR/extrinsic apoptotic pathway. Our data demonstrated that DHA combination sensitized colon cancer cells to sulindac sulfide-induced apoptosis, leading to enhanced growth suppression of human colon cancer xenografts. The combination effect was primarily attributed to increased cleavage of poly(ADP-ribose) polymerase (PARP) and caspase- 8 activation. Moreover, pretreatment with z-IETD-FMK (caspase-8 inhibitor) or stable expression of dominant negative caspase- 8 genes blocked DHA/sulindac sulfide cotreatment-induced apoptosis. In view of the finding that DR5 silencing abrogated the combination-stimulated apoptosis, we propose that apoptotic synergy induced by sulindac sulfide plus DHA is mediated via DR5. Our findings collectively support the utility of a combination of sulindac sulfide and DHA in the effective prevention and treatment of colon cancer.
\end{abstract}

\section{Introduction}

Accumulating epidemiological evidence suggests that longterm use of non-steroidal anti-inflammatory drugs (NSAIDs)

Correspondence to: Dr So Hee Kim, ${ }^{5}$ Present address: College of Pharmacy, Ajou University, Suwon, Gyeonggi-Do 443-749, Republic of Korea

E-mail: shkim67@ajou.ac.kr

Key words: docosahexaenoic acid, sulindac, apoptosis, death receptor, colon cancer reduces the risk of colorectal cancer. Sulindac is considered one of the most efficacious NSAIDs available at present, based on its ability (1) to cause regression of colonic adenomas in patients with familial adenomatous polyposis as well as its promising anticancer potential in rodents (2). However, its benefits are limited to significant, but not complete (60-70\%) reduction in the number and size of polyps, and clinical trials have further disclosed various side effects, such as gastrointestinal and renal toxicity after long-term use. Combination of another chemopreventive and anticancer agents with sulindac may provide an ideal strategy to enhance the efficacy of sulindac and eliminate unwanted side effects in preclinical and clinical studies by lowering effective doses. With this regard, a recent randomized controlled clinical trial showed that a lowdose combination of sulindac and difluoromethylornithine, an inhibitor of ornithine decarboxylase, reduces the risk of recurrent colorectal adenomas and high-risk adenomas by 70 and $92 \%$, respectively, confirming the potential of combination chemoprevention strategies including sulindac (3).

Sulindac and its analogs appears to exert chemopreventive and anticancer effects via its apoptosis-inducing capability (4), although the underlying mechanisms remain to be established. Sulindac sulfide, the major active metabolite of sulindac, induces apoptosis in a wide range of cancer cells like the parent compound sulindac, but at much lower doses compared with sulindac. Sulindac sulfide triggers apoptosis through the mitochondrial pathway involving caspase-9 (5). In addition, the compound upregulates death receptor (DR5) expression at both the mRNA and protein levels and activates caspase- 8 in prostate and colon cancer cell lines. Moreover, sulindac sulfide cooperates with Apo2L/TRAIL, the ligand for DR, to induce apoptosis (6). Therefore, it appears that sulindac sulfide is a compound that triggers DR5-dependent extrinsic apoptotic pathway at least in certain types of cancer cells (2).

Induction of apoptosis via activation of the DR-mediated extrinsic apoptotic pathway may represent an ideal therapeutic strategy to eliminate cancer cells since the pathway trigger apoptosis in a wide range of cancer cells while minimally affecting normal cells (7). Activation of DR can be achieved with either ligand binding or receptor clustering and aggregation (8). Many of the currently used chemotherapeutic drugs are inducers of DR expression. Therefore, anticancer agents 
that act lower the sulindac dose to induce DR-mediated apoptosis may provide effective and safe combination regimens with sulindac.

Diets rich in omega-3 polyunsaturated fatty acids (PUFAs) reduce the incidence and growth of colorectal cancer. Consistent with these epidemiological observations, laboratory studies have shown that omega-3 PUFAs suppress the formation and growth of colorectal cancer in both established cell lines and animal models. One of the suggested underlying anticancer mechanisms is the induction of apoptosis by PUFA (9). The extensively characterized docosahexaenoic acid (DHA) is more potent at inducing apoptosis than other omega-3 PUFAs, such as eicosapentaenoic acid (10). DHA reduces viability and triggers apoptosis in colon cancer cells while increasing the viability of normal cells $(11,12)$, and is additionally effective in enhancing the chemosensitivity of cancer cells when administered in combination with chemotherapeutic drugs, such as doxorubicin and irinotecan (13-15). Recent studies have further demonstrated that DHA modifies the expression of key molecules critical in apoptosis, including $\beta$-catenin, survivin and XIAP $(9,16)$. DHA activates caspase- 8 and downregulates FLIP, an endogenous inhibitor of DR-mediated apoptotic signaling, in colon cancer (16), and sensitizes colon cancer cells to Fas-mediated apoptosis (17). Moreover, DHA activates procaspase- 8 , but not procaspase-9 (10). The compound cooperates with TRAIL, a DR ligand, to induce apoptosis in HT-29 colon cancer cells (18). It is hypothesized that DHA may act in concert with sulindac sulfide to promote extrinsic apoptotic signaling mediated by DR. To examine this possibility, we investigated the potential of a combination of DHA and sulindac sulfide in inducing growth suppression and apoptosis in colon cancer.

\section{Materials and methods}

Cell lines and cultures. The human HCT-116 and LoVo colon cancer cell lines were purchased from the American Type Culture Collection (ATCC, Manassas, VA, USA), and the human SNU-C4 cell line was purchased from the Korean Cell Line Bank (Seoul, Korea). Cells were cultured in RPMI-1640 medium supplemented with $10 \%$ heat-inactivated fetal bovine serum (FBS) (Hyclone, Logan, UT, USA) and 100 units/ml each of penicillin and streptomycin. All cells were grown in incubators in a humid atmosphere of $95 \%$ air $/ 5 \% \mathrm{CO}_{2}$.

Reagents and antibodies. DHA, 3-(4,5-dimethylthiazol-2-yl)2,5-diphenyl-tetrazolium bromide (MTT) and propidium iodide were obtained from Sigma (St. Louis, MO, USA). Sulindac sulfide was obtained from LKT Laboratories (St. Paul, MN, USA). z-VAD-FMK (pan-caspase inhibitor), z-IETD-FMK (caspase- 8 inhibitor) and z-LEHD-FMK (caspase-9 inhibitor) were obtained from Enzyme Systems Products (Livemore, CA, USA). Ac-IETD-AFC (caspase-8 substrate) was purchased from Alexis Biochemicals (Lausen, Switzerland). Antibodies to human poly(ADP-ribose) polymerase (PARP) and Bid were purchased from BD Pharmingen (San Diego, CA, USA) and antibodies to procaspase- 8 and -9 were obtained from Cell Signaling Technologies (Beverly, MA, USA). Antibodies to $\beta$-actin were obtained from Sigma. Antibodies to death receptor-5 (DR-5) and TRAIL were obtained from Alexis
Biochemicals. All other chemicals were of reagent grade and used without further purification.

Cell viability analysis. Cells were seeded into 96-well plates. Starting $24 \mathrm{~h}$ later, cells were treated with various doses of sulindac sulfide or DMSO vehicle in the presence or absence of DHA. At $48 \mathrm{~h}$ post-incubation, the growth and viability of cells were determined by using MTT. The ability of cells to form formazan crystals by active mitochondrial respiration was determined using a microplate spectrophotometer (BioTek, Winooski, VT, USA) after dissolving the crystals in DMSO on the shaker (IKA, Staufen, Germany).

Colony formation assay. Four hundred cells were seeded in $5-\mathrm{cm}$ diameter dishes and incubated for $16 \mathrm{~h}$ at $37^{\circ} \mathrm{C}$ in an atmosphere containing $5 \% \mathrm{CO}_{2}$. Cells were then treated with increasing concentrations of sulindac sulfide in the presence or absence of DHA. The medium containing treatments was replaced every three days. After 10 days, surviving cells forming visible colonies (containing $>50$ cells) were counted after staining with crystal violet in $60 \%$ methanol.

Immunoblot analysis. At time of harvest, treated cells were scraped from the plates, washed twice with ice-cold PBS, and pelleted at $2,400 \mathrm{x}$ g for $5 \mathrm{~min}$. The cell pellet was then incubated for 15-30 min on ice in the lysis buffer containing $150 \mathrm{mM} \mathrm{NaCl}, 10 \mathrm{mM}$ Tris, $0.2 \%$ Triton X-100, 0.3\% NP-40, $0.2 \mathrm{mM} \mathrm{Na}_{3} \mathrm{VO}_{4}$ and protease inhibitors (Roche Diagnostics, Basel, Switzerland), $\mathrm{pH}$ 7.4. After centrifugation at $14,000 \mathrm{rpm}$ for $15 \mathrm{~min}$ at $4^{\circ} \mathrm{C}$, the supernatants were collected, and the protein concentration in each was measured by the Bradford method. Aliquots of supernatants containing equal amounts of protein were boiled in SDS-reducing buffer for $5 \mathrm{~min}$, electrophoresed on SDS-polyacrylamide gels, and transferred to nitrocellulose membranes. The membranes were blocked with $5 \%$ non-fat dry milk and probed with specific primary antibodies, followed by incubation with appropriate peroxidase-conjugated secondary antibodies. The blots were developed with ECL Plus reagent (Amersham, Arlington Heights, IL, USA) according to the manufacturer's protocol.

Apoptosis assay. Cytoplasmic histone-associated DNA fragments were quantified by a photometric enzyme immunoassay using Cell Death Detection ELISAplus (Roche Diagnostics) following the manufacturer's protocol. Briefly, after treatment, cells were pooled and lysed. Cytoplasmic fractions containing histone/DNA fragments were extracted and adhered to an immobilized anti-histone antibody plate. A peroxidase-conjugated anti-DNA antibody was used for detection of adhered histone/DNA fragments. A colorimetric substrate for peroxidase was then added to each well containing each sample. The net absorbances at the suggested wavelengths $\left(\mathrm{A}_{405 \mathrm{~nm}}-\mathrm{A}_{490 \mathrm{~nm}}\right)$ were obtained for the lysates derived from treated as well as control cells. Analysis was performed on a microplate spectrophotometer (BioTek).

Apoptosis induction was also assessed by flow cytometry of cells with Sub-G1 DNA content following staining with propidium iodide. For analysis of Sub-G1 DNA content cells (subdiploid cells), HCT-116 cells were plated in 60-mm cell culture dishes. After treatment, the cells were harvested 
by scraping, washed, fixed in $50 \%$ ethanol for $30 \mathrm{~min}$ and resuspended in cold-PBS. Containing $0.1 \%$ Triton X-100, $2 \mu \mathrm{l}$ of $10 \mathrm{mg} / \mathrm{ml}$ propidium iodide, and $5 \mu \mathrm{l}$ of $10 \mathrm{mg} / \mathrm{ml}$ ribonuclease A for DNA staining to be analysed by flow cytometry (FACScalibur, BD Biosciences, CA, USA). Calculation was performed with CellQuest Pro software (Becton-Dickinson, CA, USA).

Caspase activity assay. Caspase- 8 activity was determined by caspase-8 fluorometric protease assay. Cells were harvested by scraping, then washed with PBS and resuspended in lysis buffer [10 mM Tris (pH 7.4-7.5), $130 \mathrm{mM} \mathrm{NaCl}$, Triton $0.5 \mathrm{ml}$, $10 \mathrm{mM} \mathrm{NaH} \mathrm{PO}_{4}, 1 \mathrm{mM}$ EDTA, digitonin $\left.10 \mu \mathrm{M}\right]$ on ice for 15 min. Protein content was determined using the Bradford assay. The equal amount of protein were incubated with a $50 \mu \mathrm{l}$ caspase buffer [10 mM DTT, $50 \mathrm{mM}$ HEPES (pH 7.4), $1 \mathrm{mM}$ EDTA, $100 \mathrm{mM} \mathrm{NaCl}, 0.1 \%$ CHAPS] containing $100 \mu \mathrm{M}$ Ac-IETD-AFC for $2 \mathrm{~h}$ at $37^{\circ} \mathrm{C}$. The fluorescence retaining samples were analyzed by fluorescent microplate reader (XFLuor4, GENios, Haymarket, VA, USA) with a $360 \mathrm{~nm}$ excitation wavelength and a $405 \mathrm{~nm}$ emission wavelength.

Silencing of DR5 gene expression with small interfering RNA (siRNA). Gene silencing was achieved by transfecting cells with siRNAs by use of the RNAiFect transfection reagent (Qiagen, Valencia, CA, USA), following the manufacturer's instructions. DR5 siRNA duplexes that target the sequence 5'-AAGACCCTTGTGCTCGTTGTC-3', which has been reported to silence the $D R 5$ expression in other studies were purchased from Qiagen. Control siRNA oligonucleotides that target the sequence 5'-AATTCTCCGAACGTGTCACGT-3', were also employed (19). Cells plated at a density of $2.5 \times 10^{5}$ cells $/ 3 \mathrm{ml}$ of $60 \mathrm{~cm}^{2}$ dishes were transfected with DR5 siRNA or control siRNA and $48 \mathrm{~h}$ after the transfection, cells, washed with PBS, were treated with DHA/sulindac sulfide combination. On the second day after treatment, cells were harvested and subjected to apoptosis analysis. Gene silencing effects were also separately evaluated by immnunoblot analysis.

Animal studies. Five to six weeks-old female BALB/c nude mice were housed and cared for according to the standards of the Sejong University (Seoul, Korea) for animal care and were used under a protocol approved by the Sejong University on animal care. The animals were injected subcutaneously on the right flank with $1 \times 10^{7}$ human HCT-116 colon cancer cells suspended in $0.2 \mathrm{ml}$ PBS. When the tumor reached an average volume of about $25 \mathrm{~mm}^{3}$, mice were randomly divided into four groups (10-11 animals per group) and treated intraperitoneally three times a week with $500 \mu \mathrm{l}$ of vehicle, DHA alone, sulindac sulfide alone or DHA/sulindac sulfide combination. Tumors were measured using an external caliper and volume was calculated by the formula: $1 / 2 \mathrm{x}$ length $\mathrm{x}$ (width) ${ }^{2}$. All studies were performed in accordance with the approved animal protocol.

Immunohistochemistry. At the end of the experiments mice were sacrificed and the tumors were excised, fixed in $4 \%$ neutral buffered formalin and then processed for paraffin embedding and sectioning using standard histological procedures. Tumor sections were stained with hematoxylin and eosin (H\&E) for general morphologic evaluation, with anti-Ki67 antibodies (MIB-1, Dako Corp., Carpinteria, CA, USA) for proliferating cells. Briefly, the paraffin-embedded formalin-fixed tissue blocks were cut in $5 \mu \mathrm{m}$ thickness and then deparaffinized in xylene. Following a rinse with water, $3 \%$ hydrogen peroxide was applied to the slides for $4 \mathrm{~min}$ at room temperature. Another rinse with Tris-buffer was performed before the primary Ki67 ready to use antibody was overlaid for $32 \mathrm{~min}$ at $42^{\circ} \mathrm{C}$. The slides were again rinsed in Tris-buffer twice, then incubated with Biotin (iView DAB Detection Kit, Ventana Medical Systems, Inc., Tucson, AZ, USA) for $10 \mathrm{~min}$, rinsed again, and incubated with streptavidin. After a final rinse with Tris-buffer, chromogen (DAB) was applied for $8 \mathrm{~min}$ and copper solution for $4 \mathrm{~min}$. Counterstaining was then performed with commercially prepared hematoxylin for $4 \mathrm{~min}$. After the post counterstaining performed with bluing solution, the slides were dehydrated and coverslipped with Permount. Benchmark XT autostainer (Ventana Medical Systemes, Inc.) was used for all staining. Finally, the slides were observed under a light microscope at a magnification of $\mathrm{x} 400$, and photographed.

Statistical analysis. Statistically significant differences between values obtained under different experimental conditions were determined using two-tailed unpaired Student's t-tests.

\section{Results}

DHA effects on the sulindac sulfide-mediated growth inhibition. To establish the effects of DHA on sulindac sulfideinduced growth inhibition of human colon cancer cells, we treated three colon cancer cell lines, HCT-116, SNU-C4 and LoVo, with increasing doses of sulindac sulfide in the presence or absence of fixed doses of DHA. Incubation of HCT-116 and LoVo cells with $100 \mu \mathrm{M}$ sulindac sulfide alone for 2 days inhibited cell growth by 8.2 and $28.7 \%$, respectively (Fig. 1A). Notably, in the presence of $15 \mu \mathrm{M}$ DHA, $100 \mu \mathrm{M}$ sulindac sulfide inhibited HCT-116 and LoVo cell growth by 55.6 and $60.6 \%$, respectively (Fig. 1A). SNU-C4 was more resistant to the effects of sulindac sulfide than the other cell lines. Specifically, incubation of this cell line with $125 \mu \mathrm{M}$ sulindac sulfide for 2 days inhibited growth by only $0.5 \%$. In contrast, a combination of $125 \mu \mathrm{M}$ sulindac sulfide and $20 \mathrm{M} \mathrm{DHA}$ inhibited SNU-C4 cell growth by $65.0 \%$ (Fig. 1A). Incubation with DHA alone affected the growth of HCT-116, LoVo and SNU-C4 cells by $18.3,7.8$ and $16.4 \%$, respectively.

Next, we performed the colony formation assay to evaluate the combination effects of DHA and sulindac sulfide after prolonged treatment. DHA (5 $\mu \mathrm{M})$ enhanced the sulindac sulfide-induced inhibition of colony formation in HCT116 cells by 3.7- and 2.7-fold, compared with 50 and $75 \mu \mathrm{M}$ sulindac sulfide alone (Fig. 1B). Our findings collectively demonstrate that DHA sensitizes human colon cancer cells to sulindac sulfide-induced growth inhibition.

DHA effects on the sulindac sulfide-mediated apoptosis. Since the growth inhibitory effects of sulindac sulfide are known to be mediated via apoptosis, we further investigated whether DHA sensitization is associated with enhanced apop- 
A

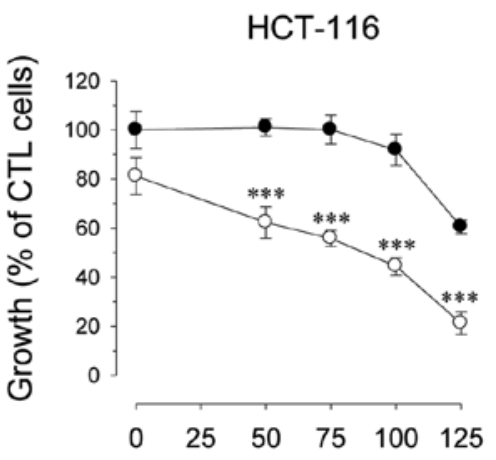

LoVo

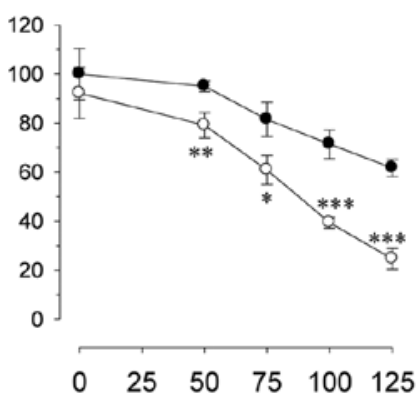

SNU-C4

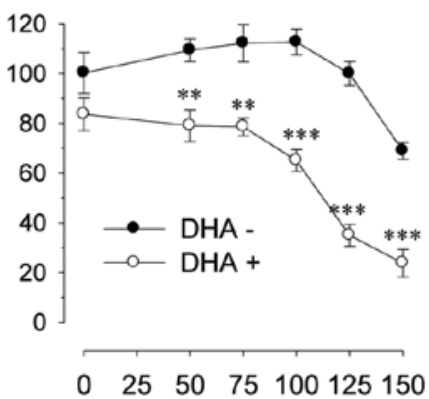

Sulindac Sulfide $(\mu \mathrm{M})$

B

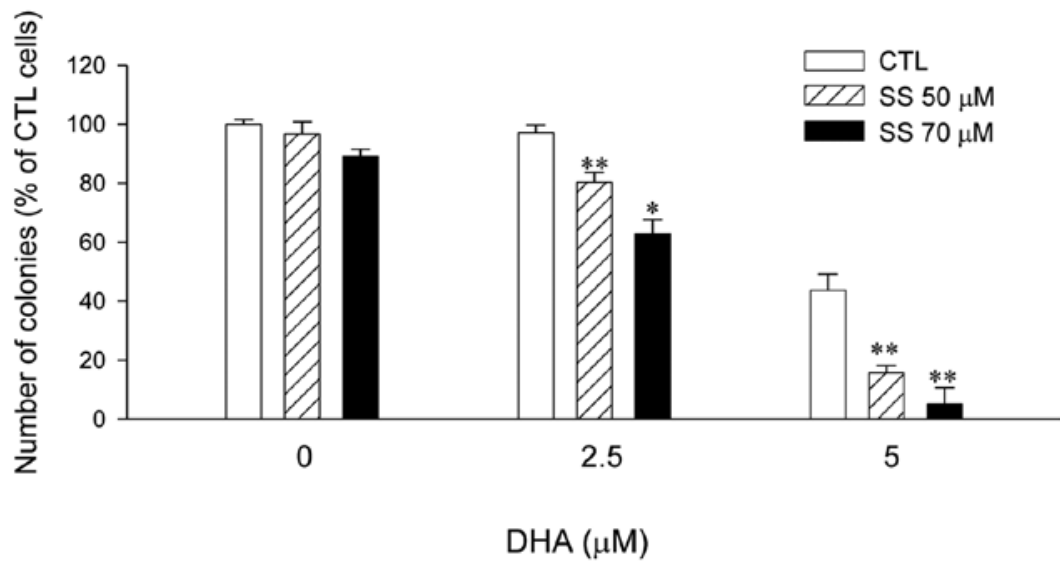

Figure 1. DHA effect on sulindac sulfide-induced growth suppression in colon cancer cells. (A) HCT-116, LoVo and SNU-C4 cells were seeded at densities

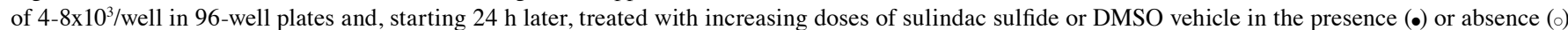
of DHA (15 $\mu \mathrm{M}$ for HCT-116 and LoVo Cells and $20 \mu \mathrm{M}$ for SNU-C4 cells). At $48 \mathrm{~h}$ post-incubation, the growth and viability of cells were determined by using MTT. Results are expressed as percentage growth (mean \pm SD of triplicate wells) relative to DMSO-treated cells. (B) Effect of combination treatment on HCT-116 colony formation. Colony formation assay was performed as described in the text. The number of colonies in the dish treated with vehicle alone was used as an index for a $100 \%$ survival rate (control) and this value was used to obtain the percentage survival rates for other dishes. Data are mean \pm SD of two independent experiments. Significant differences are indicated by asterisks: ${ }^{*} \mathrm{p}<0.05,{ }^{* *} \mathrm{p}<0.005,{ }^{* * *} \mathrm{p}<0.001 \mathrm{compared}$ with cells treated with sulindac sulfide alone. SS, Sulindac sulfide treatement.

totis. When we looked for apoptosis by the ELISA assay after treatment of HCT116 cells with $100 \mu \mathrm{M}$ sulindac sulfide in combination with $15 \mu \mathrm{M}$ DHA for $30 \mathrm{~h}$, DNA fragmentation was enhanced 2.4-fold, compared to that in cells treated with sulindac sulfide alone (Fig. 2A). DHA alone $(15 \mu \mathrm{M})$ did not increase DNA fragmentation in cells. Consistent with ELISA data, cell cycle analysis also revealed that the DHA/sulindac sulfide combination significantly enhanced the apoptotic cell content. Specifically, the proportion of cells accumulating at the sub-G1 phase was increased by $0.98-, 3.3$ - and 5.9-fold in groups treated with DHA, sulindac sulfide and DHA/sulindac sulfide, respectively (Fig. 2B). Moreover, we observed increased apoptotic morphological changes, including cell shrinkage and chromatin condensation, in cells treated with both DHA and sulindac sulfide (data not shown). Apoptosis was additionally evaluated by assaying for cleaved PARP, a general marker of apoptosis. The cleaved $85-\mathrm{kDa}$ PARP fragment was evident only when cells were treated with DHA and sulindac sulfide together for $24 \mathrm{~h}$ (Fig. 2C). Clearly, DHA enhances apoptosis induced by sulindac sulfide in human colon cancer cells.

Involvement of caspase-8 in DHA-augmented sulindac sulfide-induced apoptosis. Apoptosis is generally associated with caspase activation. Sulindac sulfide-induced apoptosis involves the caspase- 8 or -9 activation cascade, depending on the cellular context $(2,5)$. To determine whether DHA sensitization is mediated via enforced caspase activation, we examined the changes in the expression patterns of procaspase- 8 and -9 , two major initiator caspases. Expression of procaspase- 8 protein was decreased only upon DHA/sulindac sulfide co-treatment for $24 \mathrm{~h}$. On the other hand, procaspase- 9 protein expression was not affected upon sulindac sulfide treatment, regardless of the presence or absence of DHA (Fig. 3A). Caspase-8 activity was significantly increased in cells treated with the DHA/sulindac sulfide combination, as shown in Fig. 3B, consistent with immunoblot analysis data.

Since caspase- 8 activation and PARP cleavage were observed in these cell lines by DHA combination, we next examined whether pretreatment of cells with z-IETD-FMK (caspase-8 inhibitor), z-LEHD-FMK (caspase-9 inhibitor) or z-VAD-FMK (pan-caspase inhibitor) suppressed apoptotic cell death induced by DHA/sulindac sulfide. Inhibition of pancaspase and caspase- 8 blocked cotreatment-induced apoptosis to a significant extent, as evident from the marked decrease in DNA fragmentation, whereas caspase- 9 inhibition by z-LEHD-FMK pretreatment suppressed DNA fragmentation 


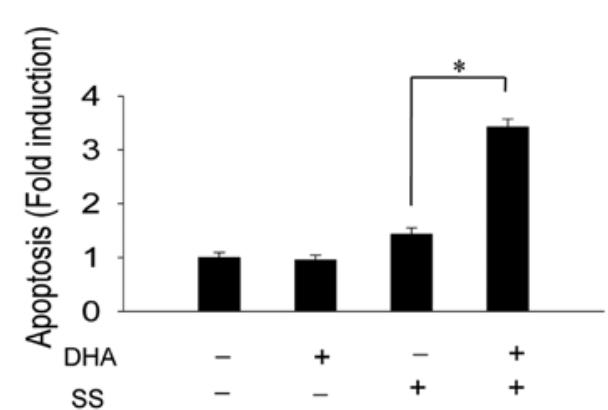

B

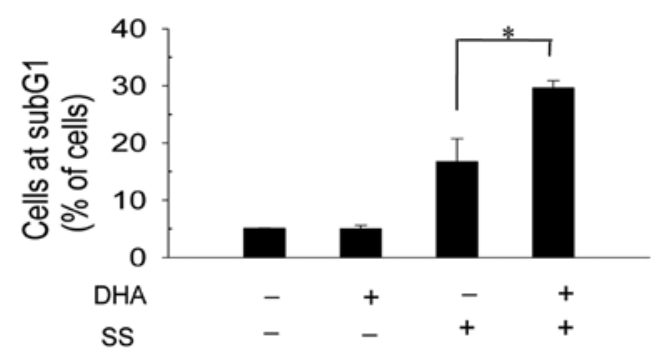

C

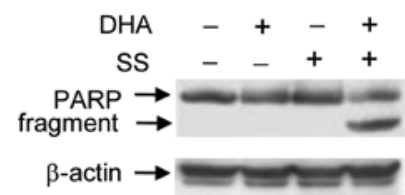

Figure 2. DHA effects on sulindac sulfide-induced apoptosis. Exponentially growing HCT-116 cells were treated with $100 \mu \mathrm{M}$ of SS in the absence or presence of DHA $(15 \mu \mathrm{M})$ for $30 \mathrm{~h}$ and then subjected to apoptosis analysis. (A) Apoptosis quantified by an ELISA that specifically detected histoneassociated DNA. Bar represents the ratio of net absorbances at the suggested wavelengths $\left(\mathrm{A}_{405 \mathrm{~nm}}-\mathrm{A}_{490 \mathrm{~nm}}\right)$ in cells treated as indicated and in control (DMSO) cells (mean $\pm \mathrm{SD}$ of three experiments performed in duplicate). (B) Percentages of cells accumulated in the sub-G1 phase analyzed as described in the text. Significant differences are indicated by asterisks: ${ }^{*} \mathrm{p}<0.05$ compared with cells treated with SS alone. (C) PARP cleavage determined by immunoblot analysis. Cell lysates obtained after $24 \mathrm{~h}$ of treatment were subjected to immunoblotting. Immunoblotting with an antibody to $\beta$-actin was used to control for equal loading of proteins per lane.

induced by the combination treatment only slightly (Fig. 3C). Similarly, in HCT116/FLICE-DN cells stably transfected with dominant-negative caspase- 8 genes (20), the extent of DNA fragmentation (Fig. 3D) and PARP fragmentation (Fig. 3E) induced by $\mathrm{DHA} /$ sulindac sulfide combination was significantly lower than that in HCT116/CTL cell lines. These results collectively suggest that DHA/sulindac sulfide-induced apoptosis is mediated by and dependent on caspase- 8 activation in HCT-116 cells.

DR5 involvement in the DHA/sulindac sulfide-mediated apoptosis. Activation of the DR with either ligand binding or receptor aggregation triggers the caspase 8-dependent extrinsic apoptotic pathway. Since sulindac sulfide appears to upregulate DR5 while having no effect on the levels of other DRs in several colon cancer cells $(2,4)$, we further examined the role of DR5 in cotreatment-induced apoptosis. At the examined doses, sulindac sulfide or DHA alone did not cause
DR5 upregulation, while a combination of sulindac sulfide and DHA enhanced DR5 expression significantly at $16 \mathrm{~h}$ post-treatment (Fig. 4A). In contrast, expression of TRAIL, the ligand for DR5, was not altered upon co-treatment. When HCT-116 cells were transfected with DR5 siRNA prior to co-treatment, DNA fragmentation was reduced by $64 \%$, compared with that in control siRNA-transfected cells (Fig. 4B). Based on these results, we propose that the DHA and sulindac sulfide combination promotes DR5 upregulation, in turn, activating caspase 8-mediated apoptotic pathways.

Treatment of HCT-116 cancer cell xenografts with the DHA-sulindac sulfide combination. Since DHA sensitized colon cancer cells to sulindac sulfide-induced growth suppression in vitro, we further examined whether DHA alters the effects of sulindac sulfide in vivo using a xenograft tumor model. For administration, DHA and/or sulindac sulfide were dispersed in distilled water using carboxymethyl cellulose and Tween-80 as the detergent mixture. Intraperitoneal administration of DHA or sulindac sulfide alone partially suppressed tumor growth, compared with that in vehicle-treated groups. Tumor size increased up to 18.6-fold from the initial day of treatment in control mice, and up to 12.0- and 10.9-fold in DHAor sulindac sulfide-treated groups, respectively. Combined administration of DHA plus sulindac sulfide suppressed tumor growth to a greater extent. The average tumor size in DHA/ sulindac sulfide-treated mice increased only by 6.9 -fold after three weeks (Fig. 5A). Consequently, the average tumor sizes in DHA-, sulindac sulfide- and DHA/sulindac sulfide-treated mice after three weeks were 0.64-, 0.57- and 0.36-fold those of vehicle-treated mice (Fig. 5B).

Immunohistochemical investigations performed at the end of the experiments revealed changes in the patterns of Ki-67 protein, a marker for cancer cell proliferation. The numbers of Ki67-positive cells were greatly reduced in sections of DHA/sulindac sulfide-treated tumors, compared with those in groups treated with DHA or sulindac sulfide alone (Fig. 2C). Together with tumor size data, the results indicate that DHA augmented sulindac sulfide-mediated inhibition of cellular proliferation in HCT-116 tumor xenografts.

We observed slight loss of body weight after 21 days of treatment in both DHA- and DHA/sulindac sulfide-treated groups, compared with vehicle- and sulindac sulfide-treated groups, but the differences were not statistically significant (data not shown).

\section{Discussion}

Recently, considerable attention has focused on the use of combinations of anticancer agents, each exerting toxicity at the optimal dose. Combination treatments may enhance the preventive or therapeutic efficacy of individual constituents and thus require lower doses of each agent. Here, we have shown for the first time that low concentrations of DHA significantly enhance growth suppression and apoptosis induced by sulindac sulfide in colon cancer cells, both in vitro and in vivo. Since pharmacologically acceptable compound DHA exerts this sensitization effect at a significantly lower dose, compared with the reported plasma concentrations $(400 \mu \mathrm{M})$ in animals fed a diet supplemented with a $5 \%$ fish oil (12), the sensiti- 


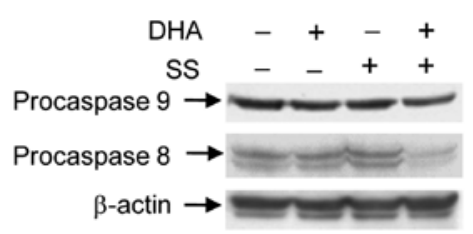

C

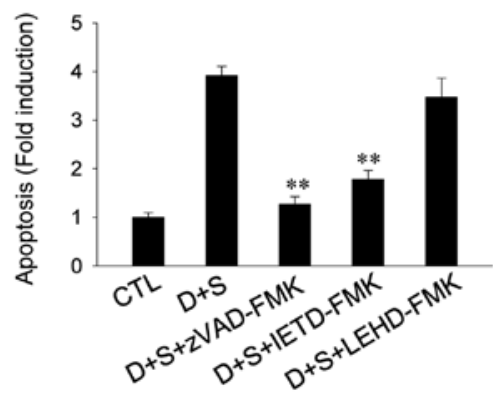

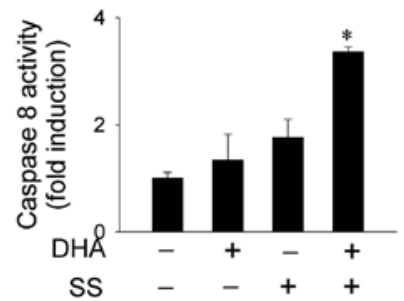

D

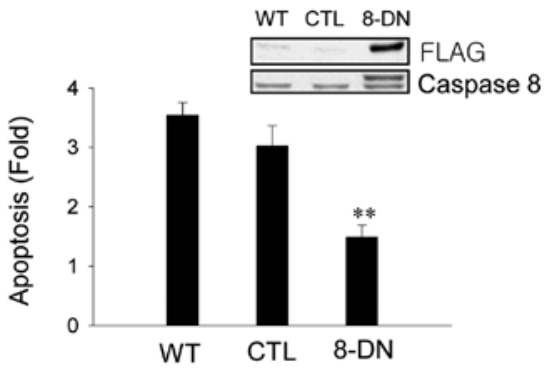

$E$

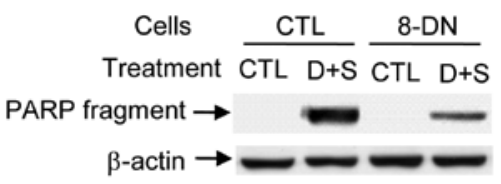

Figure 3. Involvement of caspase-8 in DHA-augmented sulindac sulfide-induced apoptosis. HCT-116 cells were treated with $100 \mu \mathrm{M}$ SS with or without $15 \mu \mathrm{M}$ DHA combination. (A) Changes in the procaspase-8 and -9 expression determined by immoblot analysis performed after 24-h treatment. (B) Effect of DHA/ SS combination on the activity of caspase-8. With cell lysates obtained after 24-h treatment, the activity of caspase-8 was measured as described in the text. Bar represents the ratio of the fluorescence in cells treated as indicated and in control cells. Data represent the average of at least three separate experiments. Significant differences are indicated by asterisks: * $\mathrm{p}<0.05$ compared with cells treated with SS alone. (C) Effect of pretreatment with z-VAD-fmk, z-IETDFMK or z-LEHD-FMK on the apoptotic cell death induced by DHA and sulindac sulfide combination. After 2-h pretreatment with $25 \mu \mathrm{M}$ of the appropriate caspase inhibitor, cells were treated with DHA/SS for $30 \mathrm{~h}$ prior to apoptosis analysis. Significant differences are indicated by asterisks: "p<0.05 compared with cells treated with DHA/SS combination. (D) Apoptosis induction by DHA/SS treatment in HCT116 cells stably transfected with empty plasmid vector (HCT116/CTL) or with plasmid containing dominant negative caspase-8 gene (HCT116/FLICE-DN). ${ }^{*} \mathrm{p}<0.05$ by unpaired t-test. (E) PARP fragmentation by DHA/SS combination in HCT116/CTL and HCT116/FLICE-DN cells.

A

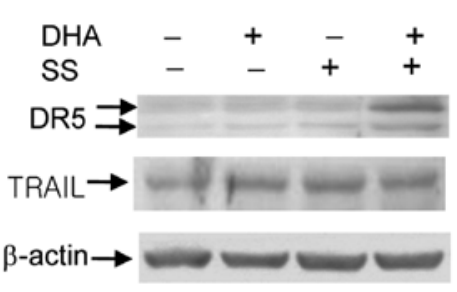

B

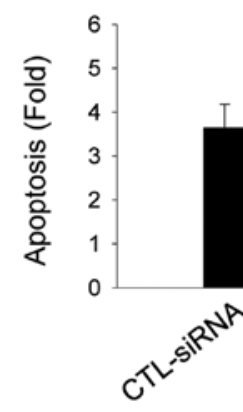

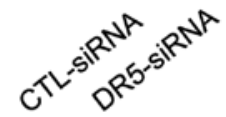

DR5

$\beta$-actin

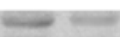

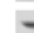

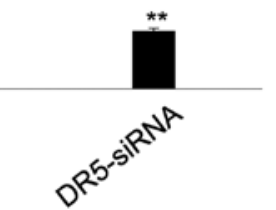

Figure 4. DR5 induction was involved in DHA/SS combination-mediated apoptosis. (A) Effect of DHA/SS combination treatment on the DR5 and TRAIL expression in HCT-116 cells. Immunoblot analysis was performed with cell lysate obtained after 16-h treatment. (B) DNA fragmentation in cells subjected to DR5 siRNA transfection prior to treatment with DHA/SS combination. Forty eight hours later after transfection with control or DR5 small interfering RNA, HCT-116 cells were treated with DMSO or $100 \mu \mathrm{M}$ sulindac sulfide $/ 15 \mu \mathrm{M}$ DHA for $30 \mathrm{~h}$. Bar represents the fold induction of DNA fragmentation, compared with DMSO-treated cells. Data are the mean value of two identical wells. " $\mathrm{p}<0.05$ compared with that of control siRNA-transfected cells treated with DHA/SS.

zation is plausible in vivo. Accordingly, the DHA/sulindac sulfide combination may provide a basis for the rational design of combinatorial strategies for the effective prevention and treatment of colon cancer and possibly other malignancies. 
A

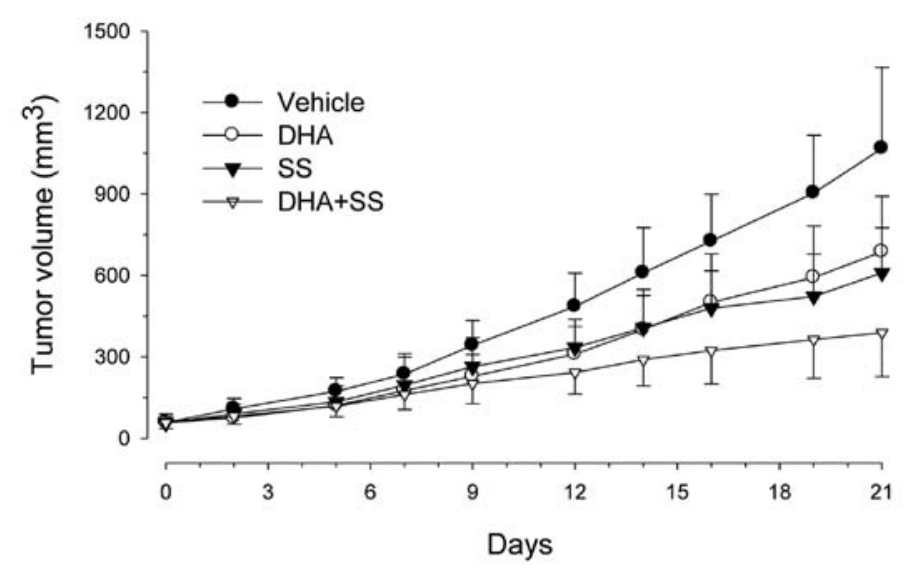

B

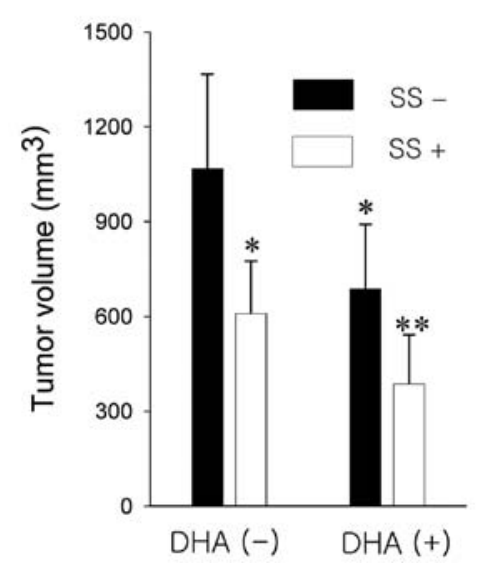

C

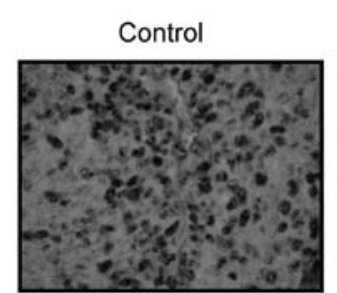

SS

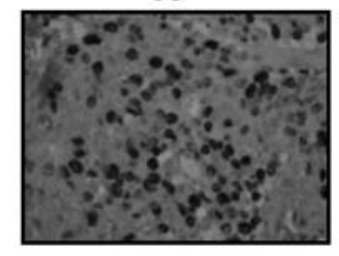

DHA

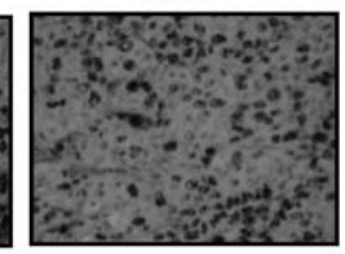

DHA+SS

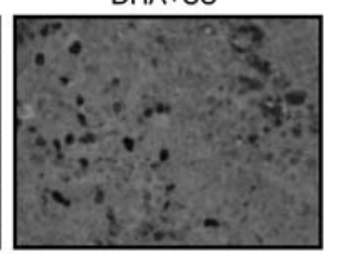

Figure 5. Sulinac sulfide in combination with DHA significantly suppressed the growth of human HCT-116 colon cancer cells implanted in nude mice, compared with those treated with sulindac sulfide or DHA alone. HCT-116 cells were xenografted on the right flank of mice as described in the text. Mice were randomly divided into four groups to receive treatment as follows: i) $500 \mu \mathrm{l}$ of vehicle ( $2 \%$ CMC and $1 \%$ Tween- 80 in distilled water); ii) $500 \mu 1$ of vehicle containing $1.1 \mathrm{mg}$ of DHA; iii) $500 \mu \mathrm{l}$ of vehicle containing $0.44 \mathrm{mg} \mathrm{SS}$; and iv) $500 \mu 1$ of vehicle containing $1.1 \mathrm{mg}$ DHA + $0.44 \mathrm{mg}$ SS. (A) Time-dependent changes in the tumor volume of the vehicle-, DHA-, SS- and DHA/SS-treated mice. (B) Tumor volume was measured on day 21 in mice. "Significant difference compared to vehicle-treated group $(\mathrm{p}<0.005)$; ${ }^{* *}$ significant difference between SS vs. DHA/SS (p<0.01). (C) Representative photographs of tumor sections of the control, DHA-, SS- and DHA/SS-treated mice. At the end of the experiments, mice were sacrificed and the tumors were excised, fixed in formalin and embedded for routine wax histology. Paraffin sections were stained with hematoxylin and eosin (H\&E) and Ki67 as a cell proliferation marker. Ki67-positive cells stain brown (original magnification, $\mathrm{x} 400$ ).

The apoptosis-inducing capability of many anticancer agents appears to underlie their chemopreventive and/or therapeutic effects. In our experiments, co-treatment with DHA significantly enhanced apoptosis of colon cancer cells induced by sulindac sulfide, leading to cooperative growth suppression of colon cancer xenografts, suggestive of improved chemopreventive or therapeutic efficacy of the two agents in combination. Since the sulindac sulfide possesses COX inhibitory activity, we were interested in establishing whether the sensitization effect of DHA was associated with increased COX inhibition. However, DHA also enhanced apoptosis induced by exisulind (sulindac sulfone), a sulindac metabolite with no COX inhibitory activity (unpublished data). The finding that the DHA sensitization effect was common to sulindac metabolites regardless of COX inhibitory activity suggests that DHA-mediated promotion of apoptosis is not associated with COX inhibition.

Earlier studies have shown that sulindac sulfide induces DR5 expression in colon cancer cells, leading to activation of the extrinsic DR5-mediated apoptotic pathway. In the present study, we observed DR5 upregulation following treatment with sulindac sulfide alone, consistent with previous reports. The DHA/sulindac sulfide combination caused upregulation of DR5 protein at doses at which neither agent alone causes such upregulation. In contrast, the expression of TRAIL, the DR5 ligand, appeared unchanged. However, increased caspase- 8 activation were concomitantly observed upon combination treatment, suggesting the involvement of an extrinsic apoptotic pathway. In this regard, it is well known that DR is activated, not only by ligand binding but also receptor clustering and aggregation (8), and thus enforced DR5 induction may trigger ligand-independent activation (21). In contrast, DR5 silencing and caspase- 8 blockade via pharmacological pretreatment with caspase-8 inhibitors or stable expression of dominant-negative caspase- 8 abrogated apoptosis induced by the DHA/sulindac sulfide combination. Therefore, it appears that enhancement of DR5 expression contributes at least partially to the apoptotic synergy of DHA/sulindac sulfide. 
While our data suggest that apoptotic synergy of the DHA/ sulindac sulfide combination is associated with augmented DR5 induction/caspase activation, the specific mechanism underlying DR5 upregulation remains unclear with our current data. Apoptosis induced by sulindac analogs requires protein kinase G-mediated activation of JNK (c-Jun $\mathrm{NH}_{2}$-terminal kinase) (22). Additional studies have reported JNK-mediated DR5 expression by specific chemotherapeutic drugs. Therefore, it is possible that DR5 induction by DHA/sulindac sulfide combination is associated with augmented JNK activation. However, further studies by our group showed that DHA does not affect the phosphorylated JNK level. Moreover, in cells pretreated with SP600125, a JNK inhibitor, DR5 upregulation by combination treatment was not affected by pretreatment (unpublished data), while sulindac sulfide alone induced JNK activation, consistent with earlier reports.

Recent studies have shown that DR5 is regulated by CCAAT/enhancer-binding protein homologous (CHOP) protein through binding to a specific site in the 5'-flanking regions of the gene $(8,23)$. Since CHOP is increased by DHA (15) as well as sulindac sulfide in colon cancers (24), we propose that DHA and sulindac sulfide act in concert to activate CHOP, resulting in augmentation of DR5 expression.

DHA is a pleiotropic molecule (13) that alters membrane structure and function and promotes formation and mobilization of ROS (25). DHA influences signal transduction of a number of pathways controlling apoptosis, for example, increasing the levels of the proapoptotic proteins, Bak and $\mathrm{Bcl}-\mathrm{xS}$, and reducing that of the antiapoptotic protein, $\mathrm{Bcl}-2$ (26). Moreover, DHA has been shown to downregulate FLIP, an inhibitor of the death receptor-mediated apoptotic pathway (16). Therefore, although not studied herein, alterations in the expression patterns of apoptosis-related proteins by DHA may also contribute to its cell death augmentation effect.

\section{Acknowledgements}

This study was supported by Basic Science Research Program through the National Research Foundation of Korea (NRF) funded by the Ministry of Education, Science and Technology (Grant 2009-0075055 to S.J. Lim and Grant 2009-0066765 to S.H. Kim).

\section{References}

1. Piazza GA, Keeton AB, Tinsley HN, Gary BD, Whitt JD, Mathew B, Thaiparambil J, Coward L, Gorman G, Li Y, Sani B, Hobrath JV, Maxuitenko YY and Reynolds RC: A novel sulindac derivative that does not inhibit cyclooxygenases but potently inhibits colon tumor cell growth and induces apoptosis with antitumor activity. Cancer Prev Res (Phila) 2: 572-580, 2009.

2. Shi J, He Q, An J, Sun H, Huang Y and Sheikh MS: Sulindac sulfide differentially induces apoptosis in Smac-proficient and -deficient human colon cancer cells. Mol Cell Pharmacol 1: 92-97, 2009.

3. Tuma R: Drugs to prevent colon cancer show promise, but hurdles remain for chemoprevention. J Natl Cancer Inst 100: 764-766, 2008.

4. Huang Y, He Q, Hillman MJ, Rong R and Sheikh MS: Sulindac sulfide-induced apoptosis involves death receptor 5 and the caspase 8-dependent pathway in human colon and prostate cancer cells. Cancer Res 61: 6918-6924, 2001.

5. Zhang L, Yu J, Park BH, Kinzler KW and Vogelstein B: Role of BAX in the apoptotic response to anticancer agents. Science 290: 989-992, 2000.
6. Sinicrope FA and Penington RC: Sulindac sulfide-induced apoptosis is enhanced by a small-molecule Bcl-2 inhibitor and by TRAIL in human colon cancer cells overexpressing Bcl-2. Mol Cancer Ther 4: 1475-1483, 2005.

7. Ohtsuki T, Kikuchi H, Koyano T, Kowithayakorn T, Sakai T and Ishibashi M: Death receptor 5 promoter-enhancing compounds isolated from Catimbium speciosum and their enhancement effect on TRAIL-induced apoptosis. Bioorg Med Chem 17: 6748-6754, 2009.

8. Elrod HA and Sun SY: Modulation of death receptors by cancer therapeutic agents. Cancer Biol Ther 7: 163-173, 2008.

9. Serini S, Piccioni E, Merendino N and Calviello G: Dietary polyunsaturated fatty acids as inducers of apoptosis: implications for cancer. Apoptosis 14: 135-152, 2009.

10. Habermann N, Schon A, Lund EK and Glei M: Fish fatty acids alter markers of apoptosis in colorectal adenoma and adenocarcinoma cell lines but fish consumption has no impact on apoptosis-induction ex vivo. Apoptosis 15: 621-630, 2010.

11. Engelbrecht AM, Toit-Kohn JL, Ellis B, Thomas M, Nell T and Smith R: Differential induction of apoptosis and inhibition of the PI3-kinase pathway by saturated, monounsaturated and polyunsaturated fatty acids in a colon cancer cell model. Apoptosis 13: 1368-1377, 2008.

12. Kang KS, Wang P, Yamabe N, Fukui M, Jay T and Zhu BT: Docosahexaenoic acid induces apoptosis in MCF-7 cells in vitro and in vivo via reactive oxygen species formation and caspase- 8 activation. PLoS One 5: e10296, 2010.

13. Biondo PD, Brindley DN, Sawyer MB and Field CJ: The potential for treatment with dietary long-chain polyunsaturated n-3 fatty acids during chemotherapy. J Nutr Biochem 19: 787-796, 2008.

14. Maheo K, Vibet S, Steghens JP, Dartigeas C, Lehman M, Bougnoux P and Gore J: Differential sensitization of cancer cells to doxorubicin by DHA: a role for lipoperoxidation. Free Radic Biol Med 39: 742-751, 2005.

15. Slagsvold JE, Pettersen CH, Storvold GL, Follestad T, Krokan HE and Schonberg SA: DHA alters expression of target proteins of cancer therapy in chemotherapy resistant SW620 colon cancer cells. Nutr Cancer 62: 611-621, 2010.

16. Giros A, Grzybowski M, Sohn VR, Pons E, FernandezMorales J, Xicola RM, Sethi P, Grzybowski J, Goel A, Boland CR, Gassull MA and Llor X: Regulation of colorectal cancer cell apoptosis by the n-3 polyunsaturated fatty acids Docosahexaenoic and Eicosapentaenoic. Cancer Prev Res (Phila) 2: 732-742, 2009.

17. Hofmanova J, Vaculova A and Kozubik A: Polyunsaturated fatty acids sensitize human colon adenocarcinoma HT-29 cells to death receptor-mediated apoptosis. Cancer Lett 218: 33-41, 2005.

18. Vaculova A, Hofmanova J, Andera L and Kozubik A: TRAIL and docosahexaenoic acid cooperate to induce HT-29 colon cancer cell death. Cancer Lett 229: 43-48, 2005.

19. Liu X, Yue P, Zhou Z, Khuri FR and Sun SY: Death receptor regulation and celecoxib-induced apoptosis in human lung cancer cells. J Natl Cancer Inst 96: 1769-1780, 2004.

20. Kim MJ, Kim SH and Lim SJ: Comparison of the apoptosisinducing capability of sulforaphane analogues in human colon cancer cells. Anticancer Res 30: 3611-3619, 2010.

21. Jang TJ, Kang HJ, Kim JR and Yang CH: Non-steroidal antiinflammatory drug activated gene (NAG-1) expression is closely related to death receptor- 4 and -5 induction, which may explain sulindac sulfide induced gastric cancer cell apoptosis. Carcinogenesis 25: 1853-1858, 2004.

22. Soh JW, Mao Y, Kim MG, Pamukcu R, Li H, Piazza GA, Thompson WJ and Weinstein IB: Cyclic GMP mediates apoptosis induced by sulindac derivatives via activation of c-Jun NH2-terminal kinase 1. Clin Cancer Res 6: 4136-4141, 2000.

23. Sung B, Ravindran J, Prasad S, Pandey MK and Aggarwal BB: Gossypol induces death receptor-5 through activation of the ROS-ERK-CHOP pathway and sensitizes colon cancer cells to TRAIL. J Biol Chem 285: 35418-35427, 2010.

24. Yang H, Park SH, Choi HJ and Moon Y: The integrated stress response-associated signals modulates intestinal tumor cell growth by NSAID-activated gene 1 (NAG-1/MIC-1/PTGF-beta). Carcinogenesis 31: 703-711, 2010.

25. Chapkin RS, McMurray DN, Davidson LA, Patil BS, Fan YY and Lupton JR: Bioactive dietary long-chain fatty acids: emerging mechanisms of action. Br J Nutr 100: 1152-1157, 2008.

26. Yamagami T, Porada CD, Pardini RS, Zanjani ED and AlmeidaPorada G: Docosahexaenoic acid induces dose dependent cell death in an early undifferentiated subtype of acute myeloid leukemia cell line. Cancer Biol Ther 8: 331-337, 2009. 